\title{
Nongeminate Radiative Recombination of Free Charges in Cation-Exchanged PbS Quantum Dot Films
}

\author{
Ashley R. Marshall, ${ }^{1,2}$ Matthew C. Beard, ${ }^{1}$ Justin C. Johnson ${ }^{1}$ \\ ${ }^{1}$ National Renewable Energy Laboratory, 15013 Denver West Pkwy., Golden CO 80401 \\ ${ }^{2}$ Department of Chemistry and Biochemistry, University of Colorado, Boulder, CO 80309
}

\begin{abstract}
:
Using photoluminescence (PL) spectroscopy we explore the radiative recombination pathways in $\mathrm{PbS}$ quantum dots (QDs) synthesized by two methods. We compare conventionally synthesized $\mathrm{PbS}$ from a $\mathrm{PbO}$ precursor to $\mathrm{PbS}$ synthesized using cation-exchange from $\mathrm{CdS}$ QDs. We show that strongly coupled films of $\mathrm{PbS}$ QDs from the cation-exchange luminesce with significant efficiency at room temperature. This is in stark contrast to conventional PbS QDs, which have exceedingly weak room temperature emission. Moreover, the power dependence of the emission is quadratic, indicating bimolecular recombination, a feature previously unreported in coupled PbS QD films. We interpret these results in terms of a greatly reduced defect concentration for cation-exchanged QDs that mitigates the influence of trap-assisted recombination. Cationexchanged QDs have recently been employed in highly efficient and air-stable lead chalcogenide QD devices, and the reduced number of trap states inferred here may lead to improved current collection and higher open circuit voltage.
\end{abstract}




\section{Keywords}

photoluminescence, $\mathrm{PbS}$, quantum dots, nanocrystals, bimolecular recombination

\section{Introduction}

One of the keys to optimal solar cell performance is to approach the so-called "radiative limit," which dictates that at open-circuit the primary recombination mechanism should be radiative decay at or very near the band gap energy.[1] For GaAs solar cells, the limit is approached due to the near-unity internal emission efficiency, and optimization of the external PL efficiency is a performance metric.[2] Although the photoluminescence (PL) of colloidal quantum dots (QDs) is often reported in order to show band gap energy, size distribution, and reasonably effective surface passivation, the emission from processed QD films relevant for solar cells is much less often reported. We have found that the PL at room temperature from a strongly coupled QD film is most often exceedingly weak $(<<1 \%)$ even in cases where the PL efficiency of the colloidal QDs is high prior to film formation (10-60\%).[3-5] We recently characterized PL in a series of PbS QD films with varying degrees of coupling and conclusively showed that the PL quantum yield (PLQY) vs. temperature directly informs about charge transport.[4] The charge carrier mobility and PLQY for most lead chalcogenide QD films are inversely related using conventional QD synthesis and film formation procedures. Photogenerated charge-carriers in coupled QD arrays can sample a large number of QDs at high temperatures, inevitably leading to strongly quenched PL even with a relatively low concentration of defects.[5]

QD solar cells are on a reasonably steep trajectory of power conversion efficiency (PCE) vs. time,[6] but the recent incremental gains involve improvements not clearly related to defect passivation, for example using structured $\mathrm{TiO}_{2}$ in order to trap light more efficiently.[7] Often, 
the fill factor (FF) appears to be the most improved feature of champion solar cells, as opposed to the open-circuit voltage $\left(V_{\mathrm{OC}}\right)$, which is far below its theoretical limit. The FF has a complicated dependence on device characteristics and can depend on factors not related to the material quality, while the $V_{\mathrm{OC}}$ is more directly influenced by defect states. In order to steepen the efficiency vs. time curve, a better defined methodology for reducing defect density would be helpful for improvements in $V_{\mathrm{OC}}$ and for enabling the use of thicker active layers that can approach nearly ideal $J_{\mathrm{SC}}$ values.

Performing ligand exchanges changes the electronic characteristics of the QDs, in some cases providing improved defect passivation and surface protection against photooxidation or other forms of degradation.[8] A variety of chemical methodologies have been used to control the QD surface chemistry, these include, but are not limited to, in situ passivation during the synthesis of the QDs, [9-12] using shorter ligands to replace native ligands that can also provide additional passivation or protection to QDs in films,[13-15] or adding a second metal chalcogenide material to encapsulate the QD in a core-shell structure. Core-shell structures have been shown to reduce the number of surface traps in lead chalcogenide QDs, revealing improved PL in solution and resulting in improved $V_{\mathrm{OC}}$ in QD devices.[16,17] The apparently reduced defect density is presumably due to replacing atoms likely to have dangling bonds with a shell material that is less prone to forming inter-gap energy states (i.e., a type-I structure). The dual benefits of reduced dangling bonds and protection from oxygen make this strategy attractive. However, a wide-bandgap shell can limit the short-circuit current in a device by confining carriers within the core due to the band offset between the core and the shell.

A modified core-shell type approach that utilizes a thin layer of lead halide instead of a metal chalcogenide has been demonstrated computationally and experimentally to be beneficial. 
Calculations suggest that a thin $\mathrm{PbI}_{2}$ layer surrounds $\mathrm{PbSe}$ QDs after iodide salt treatment, which passivates surface states and makes QDs oxidatively stable.[18] Similar results have been reported for chloride salt treatments on PbSe QDs, leaving them more air stable and with higher PLQYs than their all-organic capped counterparts.[12] These photophysical observations have translated to improved performance in recent QD devices, primarily through passivation protection from oxidation.[19] In fact, all recent record efficiency PbS QD devices have used some form of halide treatment during fabrication of the QD absorber layer.[7,9,14,19] Protection from oxidation and the removal of intrinsic surface-related inter-gap defect levels are interconnected variables in most device studies with lead chalcogenide QDs, thus the microscopic origins of improved performance remain unclear. In fact a recent study of different iodide PbS QD treatments found that the defect density was always higher in iodide treated films compared to EDT treated films.[20]

In addition to PL measurements, transient absorption (TA) spectroscopy is often used to characterize excited state lifetimes and elucidate recombination mechanisms. For isolated QDs, a model including quantum confined Auger recombination involving multiexcitons can successfully reproduce the TA data as a function of pump fluence.[21,22] For coupled QDs, the same model can be employed and produces results that reasonably reproduce the data, but important parameters, such as the biexciton lifetime, change considerably and appear to depend on the film conductivity, calling into question the validity of the exciton model. Especially problematic would be the presence of separated charge carriers within the film.[23,24] Moreover, the lifetime observed at low pump fluence, typically greater than $100 \mathrm{~ns}$ for uncoupled QDs, decreases significantly with increased QD coupling, further complicating the analysis. In many cases, there is a clear inverse relationship between the degree of QD coupling 
and the low fluence lifetime measured by time-resolved terahertz or TA. The shortened lifetime is further evidence for increased deleterious defect densities that exists after most ligandexchanges that leads to trap-assisted recombination hastened by a high charge-carrier or exciton mobility.[25,26]

In order to analyze data sets that may span large ranges of temperatures, excitation densities, and unknown strengths of inter-QD coupling (and exciton binding energies), we begin with a general model that encompasses both bulk recombination of free-charge carriers and excitonic recombination. The model includes terms that are linear, quadratic, and cubic in the excited state population. The linear terms involve geminate electron-hole radiative or nonradiative recombination and trap-assisted nonradiative decay, (often via a Shockley-Read Hall (SRH) mechanism), with rate constants $k_{X}^{r}, k_{X}^{n}$, and $k_{A}^{n}$, respectively. The quadratic terms include nongeminate electron-hole radiative recombination $\left(C_{B}^{r}\right)$ and exciton-exciton nonradiative $\left(C_{X X}^{n}\right)$ recombination coefficients. The latter term is often associated with an Auger mechanism. The cubic term describes nonradiative Auger recombination of free carriers $\left(C_{A}^{n}\right)$.[27] This model is expressed in equation 1 as a change in excited state population density $\left(N_{t}\right)$ vs. time. The superscripts on each rate constant/coefficient denote if it is radiative $(r)$ or nonradiative $(n)$.

$$
N_{t}^{\prime}=-\left(k_{A}^{n}+k_{X}^{r}+k_{X}^{n}\right) N_{t}-\left(C_{B}^{r}+C_{X X}^{n}\right) N_{t}^{2}-C_{A}^{n} N_{t}^{3}
$$

Under conditions of strong QD coupling and low fluence, the linear term involving charges $\left(k_{A}^{n}\right)$ dominates at room temperature. The other linear term involving excitons $\left(k_{X}^{r}, k_{X}^{n}\right)$ are relevant only in weakly coupled films or at low temperatures where exciton dissociation is negligible. At higher excitation density, quadratic terms become important, with the $C_{B}^{r}$ pathway commonly 
observed in high performing solar cells, including $\mathrm{PbMAI}_{3}$ perovskite films.[28] We note that the nonradiative pathway involving bimolecular charge-carrier recombination is often seen in organic solar cells with low dielectric constant (i.e., via a Langevin or related mechanism).[29] The quadratic term with coefficient $C_{X X}^{n}$ is negligible at room temperature for systems with small exciton binding energy (i.e. most bulk inorganic semiconductors). For QDs in a film, the term is only relevant when the barrier to exciton dissociation is similar to or larger than $k_{\mathrm{B}} T$. At even higher photon fluxes or in film regions where charges accumulate (i.e. at a junction under open circuit), the cubic Auger term can dominate, typically leading to fast and mobility dependent nonradiative decay.

With uncertainty about the degree to which each term in equation 1 contributes, extracting the rate constants/coefficients from a direct time-resolved measurement can be challenging. However, by measuring steady-state PL intensity vs. incident excitation intensity (proportional to $N_{\mathrm{t}}$ ), we can extract the mechanism of radiative recombination by determining the order of the kinetic process (i.e., linear or quadratic). We find that in cation-exchanged QD films the radiative recombination regime switches from linear to quadratic with increased temperature and that $\mathrm{PL}$ is not strongly quenched. This is in stark contrast to conventionally synthesized PbS QDs of the same size that show greater than two orders of magnitude quenching above $\sim 200$ K.[3] In addition, TA analysis suggests that at very low fluence within the linear recombination regime the excited state lifetime is increased significantly (i.e., $k_{A}^{n}$ is reduced) when the cationexchange method is used to synthesize the QDs. Taken together, these observations imply that greatly improved surface passivation enables carriers to undergo many more phonon-assisted tunneling events without nonradiative decay, leading to the opportunity for radiative decay at room temperature and open circuit. This improvement should have a direct impact on $V_{\mathrm{OC}}$ as 
well as the maximum thickness of QD films that can be employed in order to capture a higher fraction of available solar flux.

\section{Results and Discussion}

In Figure 1 are shown PL spectra of $3 \mathrm{~nm}$ diameter PbS QDs synthesized by cation exchange from CdS QDs, with a first

exciton at $930 \mathrm{~nm}(\sim 1.3 \mathrm{eV})$, cast

into a film on a sapphire

substrate using dip-coating with

1,2,-ethanedithiol (EDT) to

replace the native oleic acid

(OA) ligands. The room

temperature Stokes shift of $\sim 0.15$

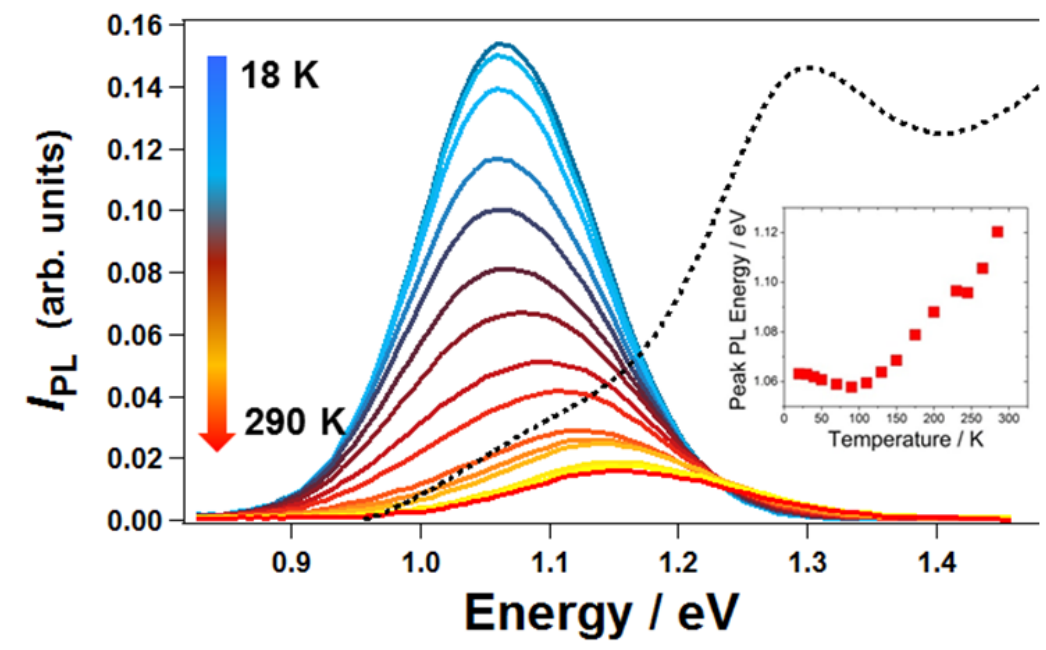

$\mathrm{eV}$ is typical for small PbS QDs

when fast energy transfer within

the distribution competes with

Figure 1: $\mathrm{PL}$ of $3 \mathrm{~nm}$ diameter PbS QDs synthesized by cation exchange, cast into a film using EDT. Dashed line is room temperature absorption spectrum. Inset: PL peak position vs. temperature.

PL. $[4,30]$ The inset highlights the peak PL energy as a function of temperature, showing an initial red shift, then a strong blue shift as the temperature increases.

The initial red-shift of the PL peak energy as the temperature is raised is indicative of band-tail behavior, $[3,31,32]$ while the general blue-shift is consistent with previous literature which shows an increase in bandgap with increased temperature.[3] More notably, the EDT-treated PbS QDs made by cation exchange show significant emission at room temperature. Strong emission at room temperature is also observed in uncoupled QDs, such as QDs capped with OA or long- 
chain thiol ligands, because the excitons are not dissociated regardless of the temperature,[4] and intra-QD excitonic radiative recombination is competitive with nonradiative recombination. However, coupled PbS QD films have previously shown 2-3 orders of magnitude of quenching at room temperature compared with $\sim 20 \mathrm{~K}$. This is thought to be due to deep trap states on the surfaces of QDs, which charge carriers readily find when they are able to sample a large number of QDs during hopping transport through the film. The reasonably strong room temperature emission of these cation-exchanged QD films suggests that the trap state density normally responsible for quenching emission has significantly decreased.

In order to prove that the QD films are not composed of isolated QDs, Fourier transform infrared spectroscopy (FTIR) measurements were performed to demonstrate a complete ligand exchange. FTIR spectra show complete removal of the OA peaks (Figure S2) to within the instrument sensitivity. Field-effect transistors (FET) were fabricated in order to further demonstrate strong electronic coupling in the films. Current-voltage scans (Figure S3) reveal an n-type film with a mobility of $3.8 \times 10^{-3} \mathrm{~cm}^{2} / \mathrm{Vs}$, which similar to or slightly higher than mobility values determined for conventional PbS QDs of a similar size $\left(2.7 \times 10^{-3} \mathrm{~cm}^{2} / \mathrm{Vs}\right.$, Table S2). Literature values of mobilities for PbS QDs treated only with short-chain organic molecules typically fall in the range $\sim 1-2 \times 10^{-3} \mathrm{~cm}^{2} /$ Vs. $[9,33,34]$ FETs are majority carrier devices and generally should be insensitive to the presence of intragap states because the high gate bias quickly fills those states and thus should not contribute to the FET measurement, but shallow defects would impact the mobility. The improvements in the FET mobility and the improved PL at room temperature suggest that the cation exchanged QDs have less mid-gap deep defects and fewer shallow defects. The n-type behavior suggests that the normally anion-rich EDT-capped PbS[35] surface has become more stoichiometric or slightly cation-rich. This may be due to residual $\mathrm{Cd}^{2+}$ from 
the cation exchange process binding to surface sulfur sites.[15] Chloride ions may also be adding electron density to the lattice if they replace surface sulfur.[9,36] Furthermore, a lower EDT packing density due to fewer available $\mathrm{Pb}^{2+}$ binding sites may reduce the density of thiolate groups at the surface that might otherwise lead to p-type behavior. The chemistry of the surface, including the locations and densities of $\mathrm{Cl}^{-}$and $\mathrm{Cd}^{2+}$, is not fully understood and requires further theoretical and experimental studies.

In Figure 2 the integrated PL across the relevant spectral region $(\sim 0.8-1.4 \mathrm{eV})$ is plotted on a log$\log$ scale as a function of incident laser power. In all cases, the power is kept far below the threshold for multiphoton absorption in a single QD $\left(<N_{\mathrm{abs}}><<1\right)$. The black squares show data for uncoupled QDs at room temperature, revealing a slope of 0.95. A slope near one is indicative of geminate recombination of the initially created electron-hole pair. At low temperature (blue circles), the slope for a coupled QD film is also $\sim 1$ due to excitonic recombination resulting from minimal thermal energy available for exciton dissociation. At room temperature (red squares), however, the slope changes to 1.9 , indicating that nongeminate bimolecular recombination of free charges, the second term in equation 1, is now dominating the radiative decay. 




Figure 2: Integrated PL intensity vs. incident laser power and calculated intensity at $514 \mathrm{~nm}$ for a film of OA-capped PbS QDs at room temperature and the same QDs after exchange of OA with EDT at two different temperatures.

Figure 3 displays the integrated PL intensity over the entire relevant spectral range vs. temperature for two samples, conventional PbS QDs and cation exchanged PbS QDs, both subsequently ligand-exchanged with EDT. The notable differences between the two curves are the slopes of PL quenching at intermediate temperatures and the strength of persistent emission as room temperature is approached. We note that there are no substantial differences in overall PLQY or temperature dependence for isolated QDs made by the different synthetic methods (data not shown). The first term in equation 2 is the previously developed model of PL quenching, derived by Zhang et al [4]. This term expresses the kinetic competition between excitonic radiative decay and exciton dissociation leading to PL quenching. We note that exciton dissociation by itself is not a decay mechanism (and thus does not appear in equation 1), but is a potential mechanism for PL quenching in the presence of defects, thus justifying its 
presence in equation 2. The model fits the conventional PbS QD PL vs. temperature well, but deviates from the cation-exchanged QD PL considerably. The second term in equation 2 is an additional pathway to radiative recombination for free charge-carriers that were born from exciton dissociation via the first term.

$$
\Phi_{P L}(T, N)=\frac{k_{X}^{r}}{k_{X}^{r}+k_{C T}(T)+k_{X}^{n}}+\Phi_{P L}^{b i}(N) \frac{k_{C T}(T)}{k_{X}^{r}+k_{C T}(T)+k_{X}^{n}}
$$

, where $k_{\mathrm{CT}}(T)$ is the exciton dissociation rate constant, expressed in equation 3 . We previously confirmed that the exciton dissociation rate constant can be equated with the temperaturedependent hopping rate constant used in dark transport measurements, thus the form follows as:

$$
k_{C T}(T)=\alpha \exp \left(-\left(\frac{T *}{T}\right)^{v}\right)
$$

, where $\alpha$ is the attempt frequency, $T^{*}$ is the characteristic temperature, and the exponent $\nu$ is related to the transport regime.[37] Here, for simplicity we have assumed $v=1$, which represents nearest-neighbor hopping.

The bimolecular radiative recombination yield $\left(\Phi_{\mathrm{PL}}^{\mathrm{bi}}\right)$ as a function of carrier density $N$ is given by:

$$
\Phi_{P L}^{b i}(N)=\frac{C_{B}^{r}}{C_{B}^{r}+{ }^{k}{ }_{A} / N+N C_{A}^{n}}
$$

which expresses the competition between rates of radiative bimolecular decay, $C_{B}^{r} N^{2}$, vs. the sum of the rates of relevant processes $\left(C_{B}^{r} N^{2}\right.$ itself, unimolecular trapping, $k_{\mathrm{A}}$, and Auger decay, $\left.C_{A}^{n} N^{3}\right)$.

Using Equation 2 to fit the data for cation-exchanged QDs, we find that the bimolecular recombination term is non-zero since the PL does not strongly quench at high temperature. The 
non-zero value for bimolecular recombination shows that carriers are able to recombine with each other radiatively before being trapped and non-radiatively decaying. We note that the term $\Phi_{P L}^{b i}$ is excitation power dependent as expected (Figure 3 inset), following a roughly quadratic dependence at low fluence before exhibiting saturation at high fluence due to the influence of higher-order nonradiative recombination (e.g., Auger decay).

In previous work on conventional PbS QDs we have demonstrated that higher mobility, correlated with higher $\alpha$ via the Einstein-Smoluchowski equation $\left(\mu=(e / 2 k T) d^{2} \alpha\right)$, leads to faster trap-assisted nonradiative recombination.[4] However, we have shown here that both the mobility and room temperature PLQY of the cation-exchanged films are higher than those of the conventional QD films, thus invalidating the previously established inverse relationship between those quantities. From the relationship between $\alpha$ and $\mu$, one might expect higher $\alpha$ for cationexchanged films; however, the opposite is true, as $\alpha$ tends to be smaller than for conventional QDs $\left(\sim 10^{8} \mathrm{~s}^{-1}\right.$ to $\left.\sim 10^{9} \mathrm{~s}^{-1}\right)$ despite the fact that the inter-QD distance $d$ should be identical. This likely reflects the fact that the films of cation-exchanged QDs are n-type instead of p-type, and thus the mobilities of holes, being the minority carriers, are probed. Hole mobilities tend to be roughly an order of magnitude smaller than electron mobilities for QDs of this size.[38] Because the parameters $\alpha$ and $\Phi_{P L}^{b i}$ have a similar effect on the shape of the quenching curves (higher $\Phi_{P L}^{b i}$ mimics lower $\alpha$ ), their influences are difficult to separate from each other. For this reason, $\alpha$ is held fixed for determination of $\Phi_{P L}^{b i}$ vs. power. More complex behavior may not be captured by the temperature-dependent PL data and thus other experiments are necessary to elucidate the full dynamical picture. 


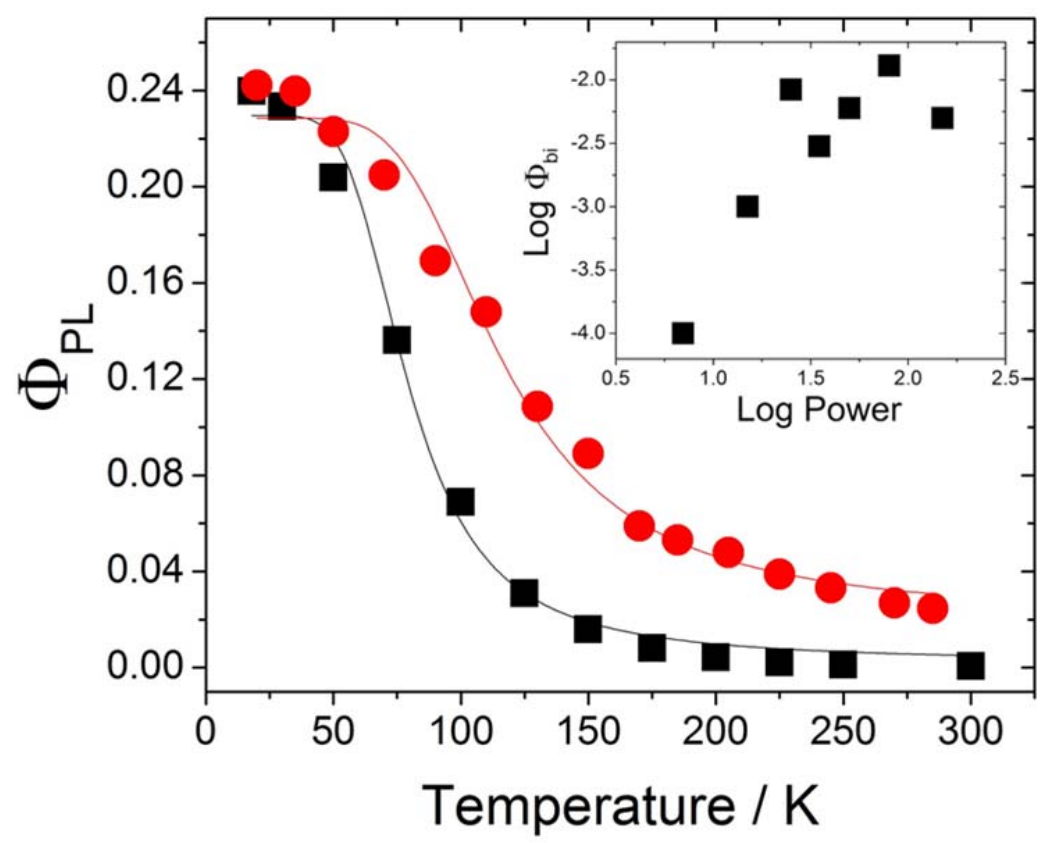

Figure 3: Integrated PL as a function of temperature for conventional PbS QDs ( $\square)$ and for cation-exchanged PbS QDs $\left({ }^{\bullet}\right)$. Inset: Log-Log plot of the power dependence of $\Phi_{P L}^{b i}$.

The transient absorption curves shown in Figure 4 were collected at various excitation densities; calculated $\left\langle N_{\mathrm{abs}}>\right.$ values are shown at the right of the figure, where $\left\langle N_{a b s}>\right.$ is calculated from the measured pump fluence and the absorption cross section.[23] A trace at similarly low $<N_{\text {abs }}>$ values is shown for an EDT-treated PbS QD film of a similar size but made by conventional synthesis (dashed curve). At the lowest excitation densities (black curves), only terms linear in the photoexcited carrier density contribute to the decay, and thus the nonradiative trap-assisted recombination term, involving $k_{A}^{n}$, dominates. The rate constant is at least one order of magnitude smaller for the cation-exchanged QDs with $\mathrm{PbCl}_{2}$ than with conventional QDs, which further underscores the reduction in deleterious trapping that occurs in the cation-exchanged QDs. We attempted to simultaneously fit the series of power dependent decay curves to a model 
of either quantum-confined Auger decay, free-carrier /bulk-like Auger decay, or a generic model including linear, quadratic, and cubic decay terms. None of these models fitted the full data set satisfactorily, although the bulk Auger decay model was a reasonable fit through most excitation densities. Inclusion of the quadratic term improved the fit slightly, but given our prior estimate of the yield of bimolecular decay (at most a few percent), it is not surprising that the fit was not greatly improved. Further investigation of the appropriate kinetic scheme for this situation is ongoing.

The TA measurements further reveal that the onset of a power dependent sub-ns decay occurs well below the $<N_{\text {abs }}>=1$ value. This is also observed in the steady-state PL power dependence, where saturation of the quadratic behavior occurs at excitation intensities of about $200 \mathrm{~mW} / \mathrm{cm}^{2}$, higher than solar intensities but far below the regime of multiple photon absorption per QD. This is likely due to diffusion assisted Auger recombination of charge-carriers, similar to that proposed by Gao et al[39] and reported in several investigations of coupled QD film dynamics.[23,25]

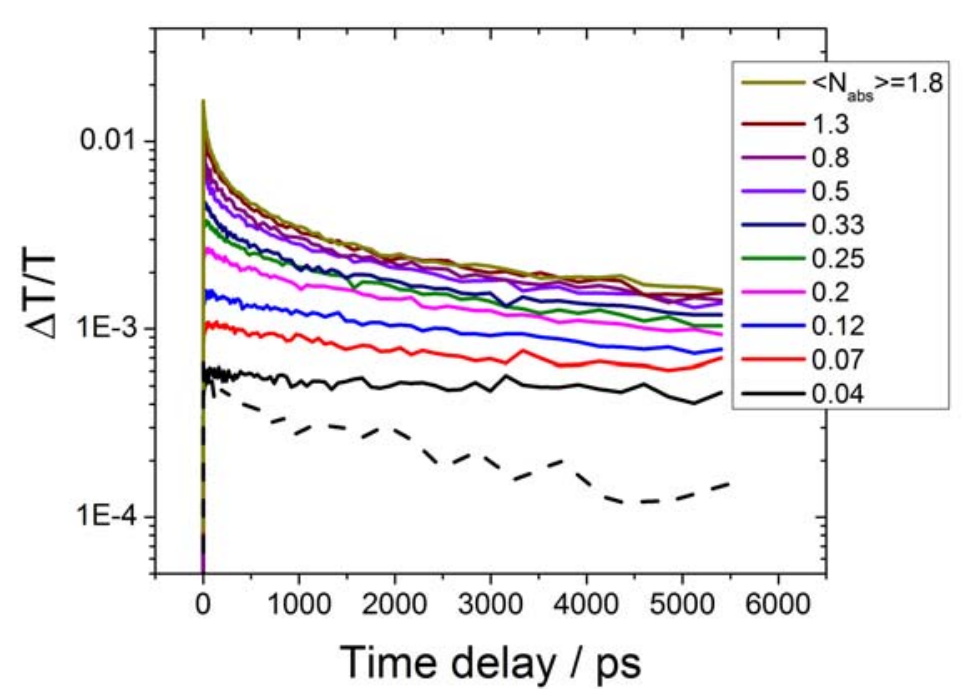

Figure 4: Transient absorption at the peak of the lowest exciton bleach signal $(\sim 930 \mathrm{~nm})$ for various excitation powers at $800 \mathrm{~nm}$ for a cation-exchanged PbS QD film. The calculated 
average exciton occupancy is shown at right. The dashed curve shows the bleach recovery for low excitation intensity of a film of conventional $\mathrm{PbS}$ QDs, also at $930 \mathrm{~nm}$.

The connection between $V_{\mathrm{OC}}$ and radiative recombination of free carriers is expressed in Equation 5:

$$
q V_{O C}=q V_{O C-I d e a l}-k T\left|\ln \eta_{\text {ext }}\right|
$$

, where $q$ is the elementary charge, $V_{\mathrm{OC}}$ is the open-circuit voltage, $V_{\mathrm{OC}-\text { Ideal }}$ is the theoretical $V_{\mathrm{OC}}$, $k$ is Boltzmann's constant, $T$ is the temperature, and $\eta_{\text {ext }}$ is the external luminescence efficiency.[1] The $V_{\mathrm{OC}}$ loss for conventionally synthesized PbS QDs would expected to be $>0.25$ V since $\eta_{\text {ext }}$ is $<10^{-4}$. For cation-exchanged $\mathrm{PbS}$ QDs, the bimolecular recombination efficiency was found to be as high as $\sim 0.02$, but at solar intensities it is $\sim 0.005$. Using $\eta_{\text {ext }}=0.005$, the $V_{\mathrm{OC}}$ loss, from Eq. 5, is around $0.14 \mathrm{~V}$, or at least $0.1 \mathrm{~V}$ smaller than with conventional QDs. It should be pointed out that this is the minimum loss because $\eta_{\text {ext }}$ is defined as only PL exiting from the front of the solar cell, which is inevitably lower than the $\eta_{\text {int }}$ measured here by reference to absolute PLQY measurements. Whereas other loss mechanisms contribute to the $\sim 0.5 \mathrm{~V} V_{\mathrm{OC}}$ deficit now commonly measured for QD solar cells, the clear observation of bimolecular radiative recombination signifies the approach of the regime achieved by other high-performing solar cells for which PL at open circuit is an important metric.

In conclusion, we have found cation-exchanged $\mathrm{PbS}$ QDs to possess a much smaller deleterious trap state density than traditionally synthesized PbS QDs. This allows coupled films of PbS QDs to emit fairly strongly (PLQY 1\%) at room temperature through bimolecular charge-carrier recombination, previously not seen in PbS QD films. The presence of halide and residual $\mathrm{Cd}^{2+}$ ions during the cation exchange process may result in improved surface defect passivation. Improving the external luminescence efficiency will increase the $V_{\mathrm{OC}}$ in devices made from $\mathrm{PbS}$ 
QD films, which has been one of the major limiting factors to increasing the efficiency in QD solar cells. Combining cation-exchanged QDs with additional surface treatments under development should lead to even better luminescence efficiency at open circuit and more efficient QD solar cells. Such PL measurements should be used as a metric for the development of new surface treatments with the caveat that the PL should scale with the light intensity as shown in Fig. 2.

\section{Experimental}

All chemicals were purchased from Sigma-Aldrich and used as received. PbS QDs were synthesized following the previously published procedure of cation exchange of CdSe to PbSe.[40] The only differences are the injection temperature and solvent. $\mathrm{PbCl}_{2}(3 \mathrm{mmol})$ was mixed with $10 \mathrm{~mL}$ oleylamine (technical grade, $95 \%$ ), degassed, and heated to $140^{\circ} \mathrm{C}$ for $30 \mathrm{~min}$. The reaction was then cooled to $90^{\circ} \mathrm{C}$ and $2.5 \mathrm{~mL}$ of previously prepared CdS QDs (366 nm first exciton, $126 \mathrm{mg} / \mathrm{mL}$ in toluene)[41] was injected. The reaction was allowed to run for 1 minute before being quenched in a water bath. The QDs were washed twice by dissolving/precipitating with the solvent/non-solvent pair hexane/ethanol. The resulting QDs were dissolved in hexane and centrifuged at $7500 \mathrm{RPM}$ for $10 \mathrm{~min}$, then filtered through a $0.2 \mu \mathrm{m}$ filter to remove any excess salts.

Films for PL and TA experiments were deposited on sapphire substrates by dipcoating with the QD in hexane solution and $1 \mathrm{mM}$ EDT in acetonitrile. The sapphire substrate was submerged in the QD solution then removed slowly. After all hexane had dried the substrate was submerged in the EDT solution for about 2 seconds and then removed slowly. This process was repeated 20-30 
times in order to build up an optically dense film with an OD 0.1-0.2 at the lowest energy exciton peak.

Pump-probe data were collected using a femtosecond transient absorption spectrometer (Helios, Ultrafast Systems). The laser source was a $4 \mathrm{~W}$ Ti-sapphire amplifier (Libra, Coherent), operating at $1 \mathrm{kHz}$ and $100 \mathrm{fs}$ pulse width. The fundamental beam at $800 \mathrm{~nm}$ was split, and a small portion $(<5 \mathrm{~mW})$ was used to generate the near-infrared continuum $(800-1700 \mathrm{~nm})$ in a 1 $\mathrm{cm}$ thick sapphire rod. Another portion was attenuated with neutral density filters and used as the excitation source after being modulated at $500 \mathrm{~Hz}$. The spot size was approximately $1 \mathrm{~mm}$ and $300 \mu \mathrm{m}$ for the pump and probe beams, respectively. The probe beam was split equally and directed to two different InGaAs array detectors. The portion that did not traverse the sample was used to cancel out laser fluctuations while the beam sent through the sample was collected and binned as either "pump on" or "pump off", depending on the timing with respect to the 500 $\mathrm{Hz}$ excitation beam. The time delay was varied with an exponential distribution of time points out to $5 \mathrm{~ns}$ and averaged for $1 \mathrm{sec}$ at each delay. Between two and ten scans were subsequently averaged. Fitting was performed in Igor Pro using a variety of kinetic schemes. The calculated $<N_{\mathrm{abs}}>$ values are determined from the pump fluence, the absorption cross section at $800 \mathrm{~nm}$, and the measured spot size.

For temperature dependent PL experiments, the films were mounted in a closed loop He flow cryostat $(18 \mathrm{~K}-325 \mathrm{~K})$ fitted with sapphire windows that was evacuated to $1 \times 10^{-4}$ Torr. The sample was cooled to $18 \mathrm{~K}$ and then heated slowly in $20 \mathrm{~K}$ steps, with an equilibration time of 10 minutes at each temperature. Collecting PL spectra during cooling instead of heating produced the same results. The $514 \mathrm{~nm}$ line of an Ar-ion laser was used to excite the sample, with a beam size of approximately $2.5 \mathrm{~mm}$ and power varying from 0.5 to $150 \mathrm{~mW}$. Each spectrum required 
roughly one minute of exposure time. The excitation beam was modulated at $500 \mathrm{~Hz}$, and the sample emission was routed through a monochromator with a 300 groove/mm grating blazed at $1250 \mathrm{~nm}$. A dual, balanced Ge photodiode was used in concert with a lock-in amplifier to detect

the signal. The spectral sensitivity of the entire system was determined with a calibrated tungsten lamp, and PL spectra were corrected accordingly.

\section{Acknowledgements}

We thank Dan Kroupa for assistance with FET sample preparation. This work was funded by the Center for Advanced Solar Photophysics, an Energy Frontier Research Center supported by the Department of Energy, Office of Science under Contract No. DE-AC36-08GO28308 with NREL.

\section{References}

[1] O.D. Miller, E. Yablonovitch, S.R. Kurtz, Strong Internal and External Luminescence as Solar Cells Approach the Shockley-Queisser Limit, IEEE J. Photovolt. 2 (2012) 303-311. doi:10.1109/JPHOTOV.2012.2198434.

[2] G. Smestad, H. Ries, Luminescence and current-voltage characteristics of solar cells and optoelectronic devices, Sol. Energy Mater. Sol. Cells. 25 (1992) 51-71. doi:10.1016/09270248(92)90016-I.

[3] J. Gao, J.C. Johnson, Charge Trapping in Bright and Dark States of Coupled PbS Quantum Dot Films, ACS Nano. 6 (2012) 3292-3303. doi:10.1021/nn300707d.

[4] J. Zhang, J. Tolentino, E.R. Smith, J. Zhang, M.C. Beard, A.J. Nozik, et al., Carrier Transport in PbS and PbSe QD Films Measured by Photoluminescence Quenching, J. Phys. Chem. C. 118 (2014) 16228-16235. doi:10.1021/jp504240u.

[5] J. Gao, J. Zhang, J. van de Lagemaat, J.C. Johnson, M.C. Beard, Charge Generation in PbS Quantum Dot Solar Cells Characterized by Temperature-Dependent Steady-State Photoluminescence, ACS Nano. 8 (2014) 12814-12825. doi:10.1021/nn506075s.

[6] Solar cell efficiency, Wikipedia Free Encycl. (2015). http://en.wikipedia.org/wiki/Solar_cell_efficiency (accessed April 8, 2015).

[7] A.J. Labelle, S.M. Thon, S. Masala, M.M. Adachi, H. Dong, M. Farahani, et al., Colloidal Quantum Dot Solar Cells Exploiting Hierarchical Structuring, Nano Lett. 15 (2015) 11011108. doi:10.1021/nl504086v.

[8] P.R. Brown, D. Kim, R.R. Lunt, N. Zhao, M.G. Bawendi, J.C. Grossman, et al., Energy Level Modification in Lead Sulfide Quantum Dot Thin Films through Ligand Exchange, ACS Nano. 8 (2014) 5863-5872. doi:10.1021/nn500897c. 
[9] A.H. Ip, S.M. Thon, S. Hoogland, O. Voznyy, D. Zhitomirsky, R. Debnath, et al., Hybrid passivated colloidal quantum dot solids, Nat. Nanotechnol. 7 (2012) 577-582. doi:10.1038/nnano.2012.127.

[10] I. Moreels, Y. Justo, B. De Geyter, K. Haustraete, J.C. Martins, Z. Hens, Size-Tunable, Bright, and Stable PbS Quantum Dots: A Surface Chemistry Study, ACS Nano. 5 (2011) 2004-2012. doi:10.1021/nn103050w.

[11] J. Tang, K.W. Kemp, S. Hoogland, K.S. Jeong, H. Liu, L. Levina, et al., Colloidal-quantum-dot photovoltaics using atomic-ligand passivation, Nat. Mater. 10 (2011) 765-771. doi:10.1038/nmat3118.

[12] J. Zhang, J. Gao, E.M. Miller, J.M. Luther, M.C. Beard, Diffusion-Controlled Synthesis of PbS and PbSe Quantum Dots with in Situ Halide Passivation for Quantum Dot Solar Cells, ACS Nano. 8 (2014) 614-622. doi:10.1021/nn405236k.

[13] D.A.R. Barkhouse, A.G. Pattantyus-Abraham, L. Levina, E.H. Sargent, Thiols Passivate Recombination Centers in Colloidal Quantum Dots Leading to Enhanced Photovoltaic Device Efficiency, ACS Nano. 2 (2008) 2356-2362. doi:10.1021/nn800471c.

[14] C.-H.M. Chuang, P.R. Brown, V. Bulović, M.G. Bawendi, Improved performance and stability in quantum dot solar cells through band alignment engineering, Nat. Mater. 13 (2014) 796-801. doi:10.1038/nmat3984.

[15] R.W. Crisp, D.M. Kroupa, A.R. Marshall, E.M. Miller, J. Zhang, M.C. Beard, et al., Metal Halide solid-State Surface Treatment for High Efficiency PbS and PbSe QD Solar Cells, Sci. Rep. 5 (2015) 9945. doi:10.1038/srep09945.

[16] M.J. Speirs, D.M. Balazs, H.-H. Fang, L.-H. Lai, L. Protesescu, M.V. Kovalenko, et al., Origin of the increased open circuit voltage in PbS-CdS core-shell quantum dot solar cells, J Mater Chem A. (2014). doi:10.1039/C4TA04785K.

[17] D.C.J. Neo, C. Cheng, S.D. Stranks, S.M. Fairclough, J.S. Kim, A.I. Kirkland, et al., Influence of Shell Thickness and Surface Passivation on PbS/CdS Core/Shell Colloidal Quantum Dot Solar Cells, Chem. Mater. 26 (2014) 4004-4013. doi:10.1021/cm501595u.

[18] J.Y. Woo, J.-H. Ko, J.H. Song, K. Kim, H. Choi, Y.-H. Kim, et al., Ultrastable PbSe Nanocrystal Quantum Dots via in Situ Formation of Atomically Thin Halide Adlayers on PbSe(100), J. Am. Chem. Soc. 136 (2014) 8883-8886. doi:10.1021/ja503957r.

[19] Z. Ning, O. Voznyy, J. Pan, S. Hoogland, V. Adinolfi, J. Xu, et al., Air-stable n-type colloidal quantum dot solids, Nat. Mater. 13 (2014) 822-828. doi:10.1038/nmat4007.

[20] F. Jähnig, D. Bozyigit, O. Yarema, V. Wood, Research Update: Comparison of salt- and molecular-based iodine treatments of PbS nanocrystal solids for solar cells, APL Mater. 3 (2015) 020701. doi:10.1063/1.4907158.

[21] V.I. Klimov, A.A. Mikhailovsky, D.W. McBranch, C.A. Leatherdale, M.G. Bawendi, Quantization of Multiparticle Auger Rates in Semiconductor Quantum Dots, Science. 287 (2000) 1011-1013. doi:10.1126/science.287.5455.1011.

[22] M.C. Beard, R.J. Ellingson, Multiple exciton generation in semiconductor nanocrystals: Toward efficient solar energy conversion, Laser Photonics Rev. 2 (2008) 377-399. doi:10.1002/Ipor.200810013.

[23] M.C. Beard, A.G. Midgett, M. Law, O.E. Semonin, R.J. Ellingson, A.J. Nozik, Variations in the Quantum Efficiency of Multiple Exciton Generation for a Series of Chemically Treated PbSe Nanocrystal Films, Nano Lett. 9 (2009) 836-845. doi:10.1021/nl803600v. 
[24] M.C. Beard, J.M. Luther, O.E. Semonin, A.J. Nozik, Third Generation Photovoltaics based on Multiple Exciton Generation in Quantum Confined Semiconductors, Acc. Chem. Res. 46 (2012) 1252-1260. doi:10.1021/ar3001958.

[25] G.W. Guglietta, B.T. Diroll, E.A. Gaulding, J.L. Fordham, S. Li, C.B. Murray, et al., Lifetime, Mobility, and Diffusion of Photoexcited Carriers in Ligand-Exchanged Lead Selenide Nanocrystal Films Measured by Time-Resolved Terahertz Spectroscopy, ACS Nano. 9 (2015) 1820-1828. doi:10.1021/nn506724h.

[26] M.E. Turk, P.M. Vora, A.T. Fafarman, B.T. Diroll, C.B. Murray, C.R. Kagan, et al., Ultrafast Electron Trapping in Ligand-Exchanged Quantum Dot Assemblies, ACS Nano. 9 (2015) 1440-1447. doi:10.1021/nn505862g.

[27] M. Ghanassi, M.C. Schanne-Klein, F. Hache, A.I. Ekimov, D. Ricard, C. Flytzanis, Timeresolved measurements of carrier recombination in experimental semiconductor-doped glasses: Confirmation of the role of Auger recombination, Appl. Phys. Lett. 62 (1993) 7880. doi:10.1063/1.108833.

[28] Y. Yamada, T. Nakamura, M. Endo, A. Wakamiya, Y. Kanemitsu, Photocarrier Recombination Dynamics in Perovskite CH3NH3Pbl3 for Solar Cell Applications, J. Am. Chem. Soc. 136 (2014) 11610-11613. doi:10.1021/ja506624n.

[29] G. Lakhwani, A. Rao, R.H. Friend, Bimolecular Recombination in Organic Photovoltaics, Annu. Rev. Phys. Chem. 65 (2014) 557-581. doi:10.1146/annurev-physchem-040513103615.

[30] L.V. Poulikakos, F. Prins, W.A. Tisdale, Transition from Thermodynamic to Kinetic-Limited Excitonic Energy Migration in Colloidal Quantum Dot Solids, J. Phys. Chem. C. 118 (2014) 7894-7900. doi:10.1021/jp502961v.

[31] J. Werner, M. Peisl, Exponential band tails in polycrystalline semiconductor films, Phys. Rev. B. 31 (1985) 6881-6883. doi:10.1103/PhysRevB.31.6881.

[32] P.T. Erslev, H.-Y. Chen, J. Gao, M.C. Beard, A.J. Frank, J. van de Lagemaat, et al., Sharp exponential band tails in highly disordered lead sulfide quantum dot arrays, Phys. Rev. B. 86 (2012) 155313. doi:10.1103/PhysRevB.86.155313.

[33] S.M. Thon, A.H. Ip, O. Voznyy, L. Levina, K.W. Kemp, G.H. Carey, et al., Role of Bond Adaptability in the Passivation of Colloidal Quantum Dot Solids, ACS Nano. 7 (2013) 76807688. doi:10.1021/nn4021983.

[34] T.P. Osedach, N. Zhao, T.L. Andrew, P.R. Brown, D.D. Wanger, D.B. Strasfeld, et al., BiasStress Effect in 1,2-Ethanedithiol-Treated PbS Quantum Dot Field-Effect Transistors, ACS Nano. 6 (2012) 3121-3127. doi:10.1021/nn3008788.

[35] B.K. Hughes, D.A. Ruddy, J.L. Blackburn, D.K. Smith, M.R. Bergren, A.J. Nozik, et al., Control of PbSe Quantum Dot Surface Chemistry and Photophysics Using an Alkylselenide Ligand, ACS Nano. 6 (2012) 5498-5506. doi:10.1021/nn301405j.

[36] W.K. Bae, J. Joo, L.A. Padilha, J. Won, D.C. Lee, Q. Lin, et al., Highly Effective Surface Passivation of PbSe Quantum Dots through Reaction with Molecular Chlorine, J. Am. Chem. Soc. 134 (2012) 20160-20168. doi:10.1021/ja309783v.

[37] P. Guyot-Sionnest, Electrical Transport in Colloidal Quantum Dot Films, J. Phys. Chem. Lett. 3 (2012) 1169-1175. doi:10.1021/jz300048y. 
[38] Y. Liu, M. Gibbs, J. Puthussery, S. Gaik, R. Ihly, H.W. Hillhouse, et al., Dependence of Carrier Mobility on Nanocrystal Size and Ligand Length in PbSe Nanocrystal Solids, Nano Lett. 10 (2010) 1960-1969. doi:10.1021/nl101284k.

[39] Y. Gao, C.S.S. Sandeep, J.M. Schins, A.J. Houtepen, L.D.A. Siebbeles, Disorder strongly enhances Auger recombination in conductive quantum-dot solids, Nat Commun. 4 (2013). http://dx.doi.org/10.1038/ncomms3329.

[40] J. Zhang, J. Gao, C.P. Church, E.M. Miller, J.M. Luther, V.I. Klimov, et al., PbSe Quantum Dot Solar Cells with More than 6\% Efficiency Fabricated in Ambient Atmosphere, Nano Lett. 14 (2014) 6010-6015. doi:10.1021/nl503085v.

[41] H. Zhang, B.-R. Hyun, F.W. Wise, R.D. Robinson, A Generic Method for Rational Scalable Synthesis of Monodisperse Metal Sulfide Nanocrystals, Nano Lett. 12 (2012) 5856-5860. doi:10.1021/nl303207s. 